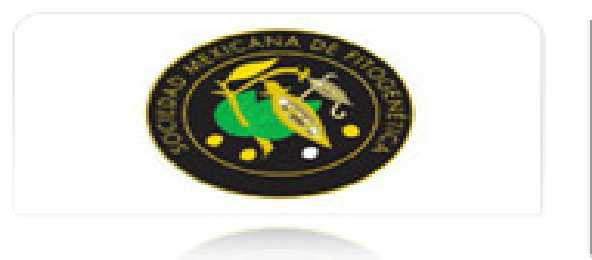

Revista Fitotecnia Mexicana

ISSN: 0187-7380

revfitotecniamex@gmail.com

Sociedad Mexicana de Fitogenética, A.C.

México

Ibarra Estrada, Emmanuel; Téllez Morales, Rocío; Soto-Hernández, Marcos; Martínez Vázquez, Mariano; García-Mateos, Rosario; San Miguel-Chávez, Rubén ACTIVIDAD ANTIMICÓTICA in vitro DE ERISOVINA Revista Fitotecnia Mexicana, vol. 32, núm. 4, octubre-diciembre, 2009, pp. 327-330 Sociedad Mexicana de Fitogenética, A.C.

Chapingo, México

Disponible en: http://www.redalyc.org/articulo.oa?id=61011789010

- Cómo citar el artículo

- Número completo

- Más información del artículo

- Página de la revista en redalyc.org

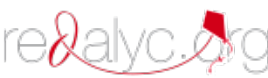

Sistema de Información Científica

Red de Revistas Científicas de América Latina, el Caribe, España y Portugal Proyecto académico sin fines de lucro, desarrollado bajo la iniciativa de acceso abierto 


\title{
ACTIVIDAD ANTIMICÓTICA in vitro DE ERISOVINA
}

\section{In vitro ANTIFUNGAL ACTIVITY OF ERYSOVINE}

\author{
Emmanuel Ibarra Estrada ${ }^{1}$, Rocío Téllez Morales ${ }^{1}$, Marcos Soto-Hernández ${ }^{1}$, Mariano Martínez Vázquez ${ }^{2}$, Rosario \\ García-Mateos $^{3}$ y Rubén San Miguel-Chávez ${ }^{*}$
}

${ }^{1}$ Posgrado en Botánica, Campus Montecillo, Colegio de Postgraduados. Km. 36.5 Carr. México-Texcoco, 56230, Montecillo, Texcoco, Edo. de México. Tel (55) 58-0459-00 Ext. 1361. ${ }^{2}$ Instituto de Química, Universidad Nacional Autónoma de México. Ciudad Universitaria, Circuito Exterior. 04510, México, D. F. ${ }^{3}$ Preparatoria Agrícola, Universidad Autónoma Chapingo. 56200, Chapingo, Edo. de México.

*Autor para correspondencia (sanmi@colpos.mx)

\section{RESUMEN}

Varios alcaloides del género Erythrina presentan actividad farmacológica que parece estar asociada con la amina terciaria espiroamina. E. americana sintetiza erisovina, alcaloide tóxico con una D L50 de $25.23 \mathrm{mg} \mathrm{kg}^{-1}$ contra ratas 'Winstar', y que no ha sido probado como agente biocida contra microorganismos. En este estudio se investigó la actividad antimicótica de la erisovina, aislada de semillas maduras de $E$. americana en los hongos fitopatógenos Alternaria solani, Botrytis cinerea, Fusarium oxysporum, Monilia fructicola, Penicillium sp. y Trichoderma harzianum mediante el método de difusión de disco de papel. $B$. cinerea, $F$. oxysporum y $M$. fructicola presentaron la mayor susceptibilidad a la erisovina, ya que una dosis de $8 \mathrm{mg} \mathrm{mL}^{-1}$ inhibió el crecimiento del micelio en 88, 57 y $43 \%$, valores superiores al testigo tratado con dimetilsulfóxido. $A$. solani, Penicillium sp. y T. harzianum, tuvieron diámetros de inhibición apenas $27 \%$ mayores que el testigo.

Palabras clave: Erythrina americana, actividad antimicótica, alcaloide, semillas.

\section{SUMMARY}

Several alkaloids of the Erythrina genus display pharmacological activity which seem to be associated with the tertiary amine spiroamine. E. americana synthesizes erysovine, a toxic alkaloid with a LD50 of $25.23 \mathrm{mg} \mathrm{kg}^{-1}$ against 'Winstar' rats, and has not been tested as an biocide agent on microorganisms. In this study the antimicotic activity of erysovine, isolated from mature seeds of $E$. americana was evaluated against phythopathogen fungi: Alternaria solani, Botrytis cinerea, Fusarium oxysporum, Monilia fructicola, Penicillium sp. and Trichoderma harzianum, using the paper disc diffusion method. $B$. cinerea, $F$. oxysporum and $M$. fructicola displayed greater susceptibility when exposed to at a dose of $8 \mathrm{mg}$ $\mathrm{mL}^{-1}$ of erysovine which inhibited mycelium by 88,57 and $43 \%$, a higher inhibition than that produced by dimethil sulfoxide used as control. In A. solani, Penicillium sp. and T. harzianum the inhibition of mycelium growth was only $27 \%$ above the control.

Index words: Erythrina americana, antifungal activity, alkaloid, seeds.

\section{INTRODUCCIÓN}

Entre los hongos fitopatógenos con importancia económica por el daño que causan a diversos cultivos, están: Alternaria solani que ocasiona la descomposición de hortalizas y frutos frescos antes y después de la cosecha (Chaerani y Voorrips, 2006); Fusarium oxysporum que perjudica a cultivos como sorgo (Sorghum bicolor L. Moench), maíz (Zea ways L.), algodón (Gossypium hirsutum L.) y alfalfa (Medicago sativa L.), así como a árboles frutales $\mathrm{y}$ forestales (Singh et al., 2007); Penicillium spp. que causan las pudriciones más comunes y a menudo las más destructivas en postcosecha (Lemmen, 1999); Monilia fructicola que provoca la pudrición café de frutos con semillas grandes (Lichou et al., 2002); Botritys cinerea que ocasiona la pudrición de frutos como fresa (Fragaria $x$ ananassa Duch) (Bautista-Baños et al., 2000); y Trichoderma harzianum, hongo oportunista que provoca pérdidas en postcosecha y presenta resistencia innata a muchos agroquímicos, como a los fungicidas.

El género Erythrina ha sido estudiado por su alto contenido en alcaloides con actividad farmacológica (García-Mateos et al., 2001). Wanjala et al. (2002) determinaron una fuerte actividad antibacteriana y antimicótica de flavonoides, pterocarpanos y de un alcaloide nuevo denominado (+)-10,11-dioxoerisotrina en E. latissima. San Miguel-Chávez y Soto-Hernández (2009) reportaron actividad antimicótica de un extracto crudo de alcaloides obtenido de plántulas de E. americana. Del mismo modo, San Miguel-Chávez et al. (2006) reportaron la presencia de erisodina, erisovina, $\alpha$-y $\beta$-eritroidina en extractos de semillas y de plántulas de $E$. americana. 
Erisovina es un alcaloide reportado como antibiótico con una DL50 de $25.23 \mathrm{mg} \mathrm{kg}^{-1}$ contra ratas 'Winstar' (Valencia et al., 1999), que es 2.5 veces más tóxico que su isómero erisodina.

En esta investigación se plantearon los siguientes objetivos: a) Extraer, purificar e identificar el alcaloide erisovina a partir de semillas de E. americana; y b) Evaluar su actividad antimicótica en contra de los hongos fitopatógenos $A$. solani, $B$. cinerea, $F$. oxysporum, $M$. fructicola, Penicillium sp. y T. harzianum.

\section{MATERIALES Y MÉTODOS}

\section{Material vegetal}

Las semillas de $E$. americana fueron recolectadas entre enero y marzo de 2004 en los jardines de la Facultad de Química de la Universidad Nacional Autónoma de México (UNAM). Se conservaron ejemplares de respaldo que se depositaron en el Herbario Nacional del Instituto de Biología, UNAM (MEXU) con el número de registro 4838 .

\section{Extracción, purificación e identificación de erisovina}

Se pulverizaron $5 \mathrm{~kg}$ de semilla, y la muestra se mantuvo en refrigeración a $5{ }^{\circ} \mathrm{C}$. Se efectuaron extracciones con hexano y posteriormente con metanol, de acuerdo con el método de Games et al. (1974). El extracto hexánico contuvo lípidos y trazas de alcaloides por lo que se descartó, y el extracto metanólico (MeOH) concentrado con alcaloides abundantes, se fraccionó por cromatografía en una columna (CC) Kimax $®$ empacada con gel de sílice G60 Merck ${ }^{\circledR}$ (70-230 mallas); para ello se colocó $1 \mathrm{~g}$ del extracto de alcaloides solubles en $\mathrm{MeOH}$ y se mezcló con sílica gel y cloruro de metileno $\left(\mathrm{CH}_{2} \mathrm{Cl}_{2}\right)$, y como eluyente se utilizó una mezcla de $\mathrm{CH}_{2} \mathrm{Cl}_{2}$ : $\mathrm{MeOH}$ cuya polaridad aumentó de 99.05:0.05 hasta 80:20.

Se recolectaron 72 fracciones de $15 \mathrm{~mL}$ cada una, las cuales se analizaron por cromatografía en capa fina, bajo las condiciones ya descritas. Se reunieron las fracciones iguales, y se obtuvo un polvo café y cristales blancos a los que se les determinó su punto de fusión con un aparato de punto de fusión Electrothermal@. Los alcaloides se purificaron por fraccionamientos sucesivos mediante $\mathrm{CC}$, con el método antes descrito; al final se recristalizó la muestra por par de disolventes (acetona/éter de petróleo) y se obtuvieron cristales amorfos a los que se les determinó su rendimiento y punto de fusión.
La identificación de la erisovina se hizo por espectroscopia ultravioleta (UV) en un espectrofotómetro Perkin-Elmer ${ }^{\circledR}$, por espectroscopia infrarroja (IR) en un espectrofotómetro Perkin-Elmer 599B $\AA$, por resonancia magnética nuclear de hidrógeno $\left(\mathrm{RMN}-{ }^{1} \mathrm{H}\right)$ y por espectrometría de masas (EM) con un aparato Varian VXR$300 \mathrm{~S} \circledast$, y su caracterización por difracción de rayos $\mathrm{X}$ en un difractómetro Bruker $\mathrm{AXS} \AA$ con detector de área $\mathrm{CCD}$ y radiación de molibdeno.

\section{Evaluación de la actividad antimicótica}

La evaluación de la erisovina como agente antimicótico se llevó a cabo mediante el método de difusión de disco (Murray et al., 1995). Se prepararon suspensiones acuosas con $10^{5}$ esporas $\mathrm{mL}^{-1}$ de los hongos Alternaria solani Sorauer, Botrytis cinerea, Fusarium oxysporum Schlechtend, Monilia fructicola L. R. Batra, Penicillium sp. y Trichoderma harzianum Rifai. La concentración de esporas en suspensión se ajustó mediante una cámara de Neubauer. Después se tomó $1 \mathrm{~mL}$ de cada suspensión, para inocular las cajas de Petri que contenían el medio de cultivo papa-dextrosa-agar. Después de 2 min se colocaron en forma equidistante siete discos de papel filtro Whatman ${ }^{\circledR}$ no. 1 de $6 \mathrm{~mm}$ de diámetro, que previamente fueron saturados con soluciones que contenían 4, 6 y $8 \mathrm{mg} \mathrm{mL}^{-1}$ de erisovina; $30 \mathrm{mg} \mathrm{mL}^{-1}$ de Captán $\quad 50 \AA \quad[(3 \mathrm{a} R, 7 \mathrm{a} S)-2-[($ triclorometil)sulfanil]3a,4,7,7a-tetrahidro- $1 H$-isoindol-1,3(2H)-diona]; $0.8 \mathrm{mg}$ $\mathrm{mL}^{-1}$ de Daconil® $(2,4,5,6$-tetracloroisoftalonitrilo ) y 0.8 $\mathrm{mg} \mathrm{mL}^{-1}$ de Benomil ${ }^{\circledR}$ (1-(butilcarbamoil) bencimidazol2-ilcarbamato de metilo); estos últimos tres son fungicidas comerciales que se utilizaron como testigos positivos.

Los fungicidas y la erisovina fueron disueltos en dimetil disulfóxido (DMSO) $0.1 \%$ y este disolvente se empleó como testigo negativo. Las cajas de Petri se incubaron a $30{ }^{\circ} \mathrm{C}$ bajo iluminación continua. Posteriormente se midió la zona de inhibición de crecimiento del micelio, la cual incluyó el diámetro del disco de papel. Las pruebas se hicieron por triplicado y con tres repeticiones. Los resultados se analizaron para cada hongo y el análisis de varianza se hizo con el modelo lineal general, y cuando hubo diferencias se aplicó la prueba de diferencia mínima significativa (DMS, 0.05). Se utilizó el paquete estadístico SAS versión 6 (SAS Institute, 1990).

\section{RESULTADOS Y DISCUSIÓN}

\section{Identificación del alcaloide utilizado}

La fracción de alcaloides liberados se separó por medio de CC sucesivas. Dado que erisovina y erisodina son 
isómeros (Figura 1), pudieron obtenerse por separado mediante lavados con acetona y metanol, y luego por recristalización por par de disolventes (acetona/éter de petróleo). Después del proceso de recristalización se obtuvo un polvo formado por cristales incoloros, con forma de prisma irregular. Los datos concernientes a la caracterización química de la erisovina fueron UV: $\lambda 285$ nm; IR: v 3500, 3080, 2842, 1185; ${ }^{1} \mathrm{HRMN}$ : 1H 6.52 (dd), 2H 5.98 (d), 3H 4.09 (m), 4Ha 1.98 (dd), 4Hb 2.52 (dd), 7H 5.68 (d), 8Ha 3.51 (m), $8 \mathrm{Hb} 3.71$ (dd), $10 \mathrm{Ha}$ $(2.461 \mathrm{~m}), 10 \mathrm{Hb} 3.47(\mathrm{~m}), 11 \mathrm{Ha} 2.07(\mathrm{~m}), 11 \mathrm{Hb} 2.92$ (m), $14 \mathrm{H} 6.61$ (l), $17 \mathrm{H} 6.84$ (s), $3 \mathrm{OCH}_{3} 3.32$ (s), 16

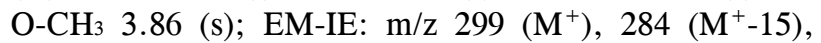
$268\left(\mathrm{M}^{+}-31\right), 215(\mathrm{M}+, 14)$. Por medio de rayos $\mathrm{X}$ en la estructura se observó un sistema diolefínico de forma casi perpendicular al sistema bencénico, cuyo ángulo diedral fue de $85.74^{\circ}$. Estos datos corroboraron la pureza del compuesto aislado, puesto que cada constante se comparó con lo reportado por Masouda et al. (1991) para este alcaloide.
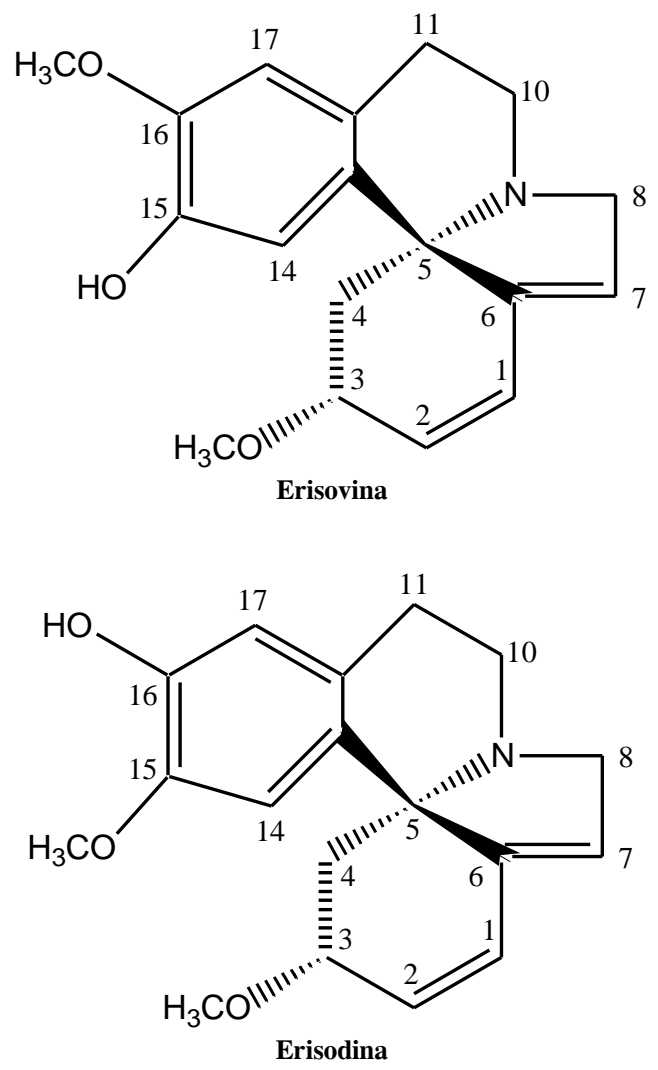

Figura 1. Estructura de los alcaloides purificados.

\section{Evaluación de la actividad antimicótica}

La erisovina demostró tener actividad antimicótica en los bioensayos llevados a cabo para tal efecto, ya que según Uzel et al. (2004) las zonas de inhibición $\geq 3 \mathrm{~mm}$ se consideran como de alta actividad. Sin embargo, se observaron diferencias en la susceptibilidad de las distintas especies fitopatógenas probadas (Cuadro 1). La actividad antimicótica de la erisovina en dosis de $8 \mathrm{mg}$ $\mathrm{mL}^{-1}$ fue mayor contra $B$. cinerea, $F$. oxysporum y $M$. fructicola, hongos en los que los diámetros de inhibición de crecimiento de micelio fueron de 88, 57 y $43 \%$ superiores al testigo con DMSO, respectivamente. La actividad contra $A$. solani, Penicillium sp. y $T$. harzianum, cuyos diámetros de inhibición apenas fueron $27 \%$ mayores que el testigo, fue menor.

Las lecturas de inhibición obtenidas con los fungicidas comerciales mostraron que el más activo fue Benomil ${ }^{\circledR}$ seguido de Captán $50 \AA$ y después de Daconil $®$. Con excepción de Penicillium sp., en las demás especies probadas la erisovina en su mayor concentración tuvo actividad antimicótica igual $(\mathrm{P} \leq 0.05)$ a la mostrada por alguno de los tres antimicóticos comerciales.

Al comparar los diámetros de inhibición de crecimiento del micelio por erisovina, con los hallados por San Miguel-Chávez y Soto-Hernández (2009) con extractos crudos de alcaloides (datos no mostrados), los primeros fueron sensiblemente menores. Esto pudiera indicar que el mayor efecto reside en un fenómeno sinergista en el cual la combinación de todos los componentes presentes en el extracto provocan un efecto superior.

En la literatura se ha reportado que la fracción de alcaloides lactónicos, como $\alpha$-y $\beta$-eritroidinas, también son sumamente tóxicos (García-Mateos et al., 2001), y éstos se encuentran en alto porcentaje en extractos de distintos tejidos de E. americana (San Miguel-Chávez et al., 2006). La actividad de erisovina contra hongos fitopatógenos no había sido reportada previamente y es comparable al alcaloide de tipo eritrinano aislado de $E$. latissima descrito por Wanjala et al. (2002), el cual también es antimicótico. Entonces, la erisovina podría ser evaluada en futuras investigaciones contra otro tipo de microorganismos, por la posibilidad de tener un mayor espectro de actividad biocida. 


\begin{tabular}{|c|c|c|c|c|c|c|}
\hline $\begin{array}{l}\text { Compuesto } \\
\text { y concentración } \\
\left(\mathrm{mg} \mathrm{mL}^{-1}\right)\end{array}$ & A. solani & B. cinerea & F.oxysporum & M.fructicola & Penicillium sp. & T.harzianum \\
\hline \multicolumn{7}{|l|}{ Erisovina } \\
\hline$(0.4)$ & sa & $7.5 \pm 1.3 \mathrm{~d}$ & sa & $7.0 \pm 0.0 \mathrm{~d}$ & sa & $7.2 \pm 0.2 b$ \\
\hline$(0.6)$ & $7.2 \pm 0.2 \mathrm{~b}$ & $8.6 \pm 0.2 \mathrm{~cd}$ & sa & $7.4 \pm 0.2 \mathrm{~d}$ & $7.2 \pm 0.2 \mathrm{c}$ & $7.0 \pm 0.0 \mathrm{~b}$ \\
\hline$(0.8)$ & $7.6 \pm 0.4 \mathrm{~b}$ & $11.3 \pm 1.8 \mathrm{bc}$ & $9.4 \pm 1.1 \mathrm{~b}$ & $8.6 \pm 0.2 \mathrm{c}$ & $7.6 \pm 0.6 \mathrm{c}$ & $7.6 \pm 0.2 \mathrm{~b}$ \\
\hline $\begin{array}{l}\text { Benomil } \\
(0.8)\end{array}$ & $7.2 \pm 0.2 b$ & $15.8 \pm 1.3 \mathrm{a}$ & $8.6 \pm 1.0 \mathrm{~b}$ & $17.4 \pm 0.2 \mathrm{a}$ & $19.6 \pm 0.5 \mathrm{a}$ & $12.2 \pm 0.4 \mathrm{a}$ \\
\hline $\begin{array}{l}\text { Captan } 50 \\
(30)\end{array}$ & $13.0 \pm 0.9 \mathrm{a}$ & $12.8 \pm 0.6 \mathrm{ab}$ & $16.0 \pm 0.5 \mathrm{a}$ & $13.8 \pm 0.4 \mathrm{~b}$ & $13.2 \pm 0.4 \mathrm{~b}$ & $7.2 \pm 0.2 \mathrm{~b}$ \\
\hline $\begin{array}{l}\text { Daconil } \\
(0.8)\end{array}$ & $7.6 \pm 0.2 \mathrm{~b}$ & $11.2 \pm 1.5 \mathrm{bc}$ & $7.6 \pm 0.6 \mathrm{~b}$ & $8.6 \pm 0.2 \mathrm{c}$ & $14.6 \pm 2.9 \mathrm{~b}$ & $7.6 \pm 0.2 \mathrm{~b}$ \\
\hline
\end{tabular}

Medias con letras iguales en una columna no fueron significativamente diferentes (DMS, 0.05). El diámetro del testigo fue $6.0 \mathrm{~mm} . \mathrm{sa}=$ Sin actividad

\section{CONCLUSIONES}

La erisovina posee un efecto inhibitorio en el crecimiento micelial de los hongos fitopatógenos $A$. solani, $B$. cinerea, F. oxysporum, Penicillium sp. y T. harzianum. La dosis de 8 $\mathrm{mg} \mathrm{mL}^{-1}$ fue la que presentó la actividad antimicótica más alta. Penicillium $s p$. fue la especie sobre la cual la erisovina tuvo su menor efecto. Erisovina es potencialmente aceptable para llevar a cabo otros estudios biodirigidos con el fin de probar su efectividad contra otra clase de microorganismos, incluso parásitos del ser humano.

\section{AGRADECIMIENTOS}

Los autores agradecen al Laboratorio de Hongos Fitopatógenos del Colegio de Postgraduados y en especial a la $\mathrm{M}$. C. Victoria Ayala Escobar, por la donación de las cepas de hongos con que se trabajó.

\section{BIBLIOGRAFÍA}

Bautista-Baños B S, M Hernández-López, L L Barrera-Necha (2000) Antifungal screening of plants of the State of Morelos, Mexico against four fungal postharvest pathogens of fruits and vegetables. Rev. Mex. Fitopatol. 18:36-41.

Chaerani R, R E Voorrips (2006) Tomato early blight (Alternaria solani): the pathogen, genetics and breeding for resistance. J. Gen. Plant Pathol. 72:335-347.

Games D E, A H Jackson, N A Khan, D S Millington (1974) Alkaloids of some African, Asian, Polynesian and Australian species of Erythrina. Lloydia 37:581-588.

García-Mateos R, M Soto-Hernández, H Vibrans (2001) Erythrina americana Miller ("Colorín"; Fabaceae), a versatile resource from Mexico: a review. Econ. Bot. 55:391-400.

Lemmen C (1999) Moulds: Occurrence, Health Risks, Protective Measures. Springer Verlag. Berlin, Alemania. 192 p.

Lichou J, J F Mandarin, D Breniaux, V Mercier, P Giauque, D Desbrus, P Blanc, E Bellau (2002) Monilia diseases on fruit trees. The emergence of a new species: Monilia fructicola. Infos-Ctifl 179:32-36

Masouda E A, M Shamma, A J Freyer (1991) The tetracyclic Erythrina alkaloids. J. Nat. Prod. 54:329-363.
Murray P R, E F Baron, M A Pfaller, F C Tenover, R H Yolke (1995) Manual of Clinical Microbiology 6. ASM, Washington DC, USA. 1005 p.

San Miguel-Chávez R, M Soto-Hernández (2009) Chemical composition and antifungal activity of the alkaloid extract of Erythrina americana Miller seedlings. J. Agric. Food Chem. (En prensa).

San Miguel-Chávez R, M Soto-Hernández, T Terrazas, G Kite (2006) Morphology and alkaloidal profile of the seedlings of Erythrina americana Mill. and E. coralloides A. DC. Feddes Repert. 117:232-239.

SAS Institute (1996) SAS/ STAT Guide for Personal Computers, Version 6.12. SAS Institute, Cary, N.C. 1028 p.

Singh G, W Chen, D Rubiales, K Moore, Y R Sharma, Y Gan (2007) Diseases and their management. In: Chickpea Breeding and Management. S S Yadav, R J Redden, W Chen, B Sharma (eds). CABI International. pp:497-519.

Uzel A, A Guvensen, E Cetin (2004) Chemical composition and antimicrobial activity of the essential oils of Anthemis xylopoda $\mathrm{O}$. Schwarz from Turkey. J. Ethnopharmacol. 95:151-154.

Valencia G T, M Soto-Hernández, J A García, E M Servín (1999) Determinación de la dosis letal media ( $\mathrm{LD}_{50}$ ) y caracterización química preliminar de fracciones de alcaloides de Erythrina herbacea. In: Productos Naturales. J A Lechuga, F Cruz (eds). Perspectivas Biotecnológicas. Vol. IV. Universidad Autónoma Metropolitana, Unidad Iztapalapa, México, DF. pp:67-75.

Wanjala C C W, B F Juna, B A Bojase, R R T Majinda (2002) Erythrinaline Alkaloids and Antimicrobial Flavonoids from Erythrina latissima. Planta Médica 68:640-642. 